\title{
Haemodialysis Cost Reduction by Artificial Kidney Storage : a Simple, Effective Technique for Re-use of Coil Kidneys
}

\author{
R. P. BELL * ; J. E. FIGUEROA,† M.D.
}

British Medical fournal, 1970, 1, 788-789

Cummary: A simple method of storing and re-using coil $\checkmark$ dialysers was used in over 700 dialysers without serious complications but mantaining the efficiency of the dialyser. The method can reduce the expense of coil and tubing by as much as $\mathbf{7 0} \%$, while the total cost of home or hospital dialysis can be reduced by as much as 15 to $25 \%$ even when the coil is re-used only once. Coils were re-used as many as 14 times, making the potential reduction in cost much larger. This technique deserves further evaluation in both hospital and home dialysis programmes.

\section{Introduction}

Haemodialysis is now a well-established method of treatment for chronic uraemia. Since the introduction of the twin-coil kidney by Kolff and Watschinger (1956), this dialyser has been widely used in the treatment of acute and chronic renal failure. Several groups have tried to reduce the great expense of chronic dialysis by using the coil more than once in the same patient. The methods described in the literature, though effective, appear complicated and at times serious complications have resulted. We describe a simple and safe method of storing and re-using coil dialysers which is applicable to both hospital and home dialysis.

\section{Material and Method}

The 100-litre tank supplied by Travenol Laboratories with double canisters was employed in all dialyses. A warm dialysate was used, with dialysate bath changes every two hours. The Twin Coil 190, Ultra Flow 145, Ultra Flow 100, and Chron-A-Coil 90 were all utilized in this study. All coils were stored and re-used as many times as possible, until leakage occurred or a febrile reaction developed in the patient. Evidence of sepsis in the patient before or at the time of dialysis was regarded as a contraindication to coil re-use.

At the completion of dialysis the blood was removed from the coil by air displacement. This blood was either reinfused to the patient while being displaced by air or stored by one of the methods shown in Fig. 1. Heparin was added to the blood $(5,000$ units) whenever the coil and blood were stored together. Recirculation of this blood and storage were performed as previously described (Shaldon et al., 1964; Tchetchik et al., 1966).

After removing the blood by air displacement, two to four litres of heparinized sterile saline was circulated through the coil, continuing until the return from the venous side of the dialyser was free of blood and fibrin particles (Fig. 2). The arterial and venous anastomoses were then connected by means of a sterile silicone rubber sleeve, and all other openings were closed with sterile plastic plugs. The 100-litre tank was then drained, washed, and partially filled with cold tapwater. The cold tap-water was recirculated through the

\footnotetext{
* Senior Medical Student, L.S.U. Medical School; Summer Clinical Research Fellow, Alton Ochsner Medical Foundation.

† Chairman, Dialysis and Renal Transplantation Program, Ochsner Medical Center, Department of Internal Medicine, Section I, Ochsner Clinic, New Orleans, Louisiana 70121.
}

outside of the coil kidney for 10 minutes. The coil was then removed carefully and stored in a clean plastic bag at $4^{\circ} \mathrm{C}$. until re-use. Antibiotics, antiseptic solutions, and added heparin were not utilized in this technique.

At the time of the next dialysis the coil was placed in the tank in the usual manner, again recirculating tap-water on the outside for 10 minutes, followed by a two- to four-litre rinse with heparinized sterile saline to remove further particles from the blood compartment. Dialysis was then started by displacing the saline with the patient's own blood. A leakage test was done before each re-use, utilizing 5 to $10 \mathrm{ml}$. of blood during the saline rinse. Whenever leakage of blood occurred during dialysis, reinfusion of the blood from the coil to the patient was attempted in all cases, unless the leakage was large enough to prevent displacement of blood.

\section{Results}

Sixty-eight patients have received over 700 dialyses on re-used coils. During the three months March to May 1969166 dialyses were done at Ochsner Foundation Hospital. During this time 38 coils were used more than once, 120 dialyses being carried out with them. This gives a ratio of 3.2 dialyses per coil. There were no serious complications attributable to the re-use of coils. Febrile reactions were seen only rarely, and in no case were they associated with bacteraemia, as con-

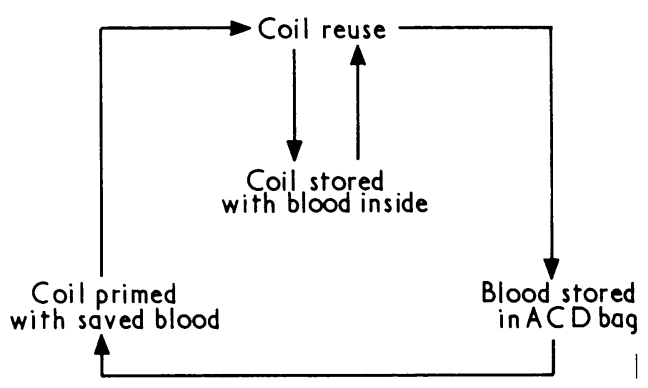

FIG. 1.-Alternatives for storage of blood from the coil dialyser.

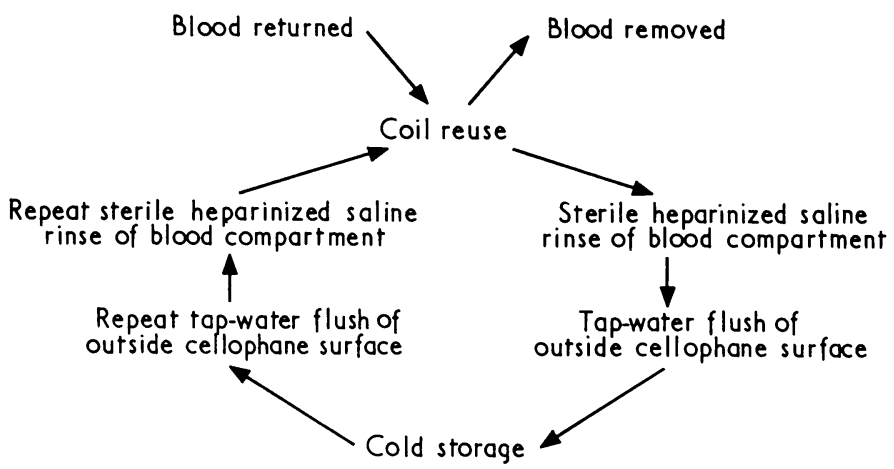

Fig. 2.-Schematic representation of the technique for storage of the coil dialyser. 
firmed by multiple blood and saline cultures. Coils have been re-used as many as 14 times without complications and without appreciable drop in dialysance of urea. These results corroborate previous reports (Johnson et al., 1969). It takes an experienced person about 15 minutes to prepare the coil for storage and an equal period of time to set it up for re-use.

\section{Comments}

Storage and re-use of coils can be done very effectively and easily. This method can be used in home dialysis and would considerably reduce the cost of chronic dialysis. In our experience the expenditure for new coils and tubing can be reduced to at least one-third simply by the re-use of coils. This technique is limited only by the occurrence of leaks, clotting within the coil, and sepsis. Contrary to previous reports, we have not found it necessary to use antibiotics or antiseptic solutions as prophylaxis against infection (Tchetchik et al., 1966; Johnson et al., 1969). Reinfusion of blood to the patient at the end of each dialysis has eliminated the problem of haemolysis in stored coil blood. We have rinsed the outside of the coil with cold tap-water to reduce bacterial growth and toxin formation on the outside of the membrane.

No haemorrhagic diatheses have been seen with re-use of coils in our experience, in contrast with a previous report (Tchetchik et al., 1966). With this technique the immediate postoperative period is not a contraindication to re-use of the coil (Tchetchik et al., 1966) because the stored coil contains only a small amount of heparin (500 to 1,000 units). Even regional heparinization can be performed with coils stored in the manner described.

\section{REFERENCES}

Johnson, C. E., et al. (1969). Fournal of the American Medical Association, 207, 2087.

Kolff, W. J., and Watschinger, B. (1956). Fournal of Laboratory and Clinical Medicine, 47, 969.

Shaldon, S., Silva, H., and Rosen, S. M. (1964). British Medical fournal, 2,411 .

Tchetchik, M., Nakamoto, S., and Kolff, W. J. (1966). Fournal of the American Medical Association, 196, 451.

\title{
Clinical Associations of II-Hydroxycorticosteroid Suppression and Non-suppression in Severe Depressive Illnesses
}

\author{
B. J. CARROLL,* M.B., B.SC., D.P.M. ; BRIAN DAVIES, † M.D., M.R.C.P., D.P.M., F.A.N.Z.C.P.
}

Cummary: Fifty patients with severe depression were separated into two groups by the responses of their plasma 11-hydroxycorticosteriod levels to a midnight dose of $2 \mathrm{mg}$. of dexamethasone. Clinical and questionary comparisons were made between the two groups of patients, who were similar as regards age, sex, and length of symptoms before admission to hospital. No differences were found between the groups of severely ill patients as regards the severity of their depression and anxiety assessed by questionary. Nevertheless, agitation was significantly greater in the patients whose corticosteroid levels were not suppressed by dexamethasone and adverse childhood experiences in those whose levels were suppressed.

\section{Introduction}

Carroll et al. (1968) showed that the plasma 11-hydroxycorticosteroid (11-OHCS) response to a midnight 2-mg. dose of dexamethasone phosphate was abnormal in 14 out of 27 severely depressed patients. Patients in the same ward with other psychiatric illnesses, and the same depressed patients on recovery, did not show this abnormality. Another test of hypothalamic function-the plasma 11-OHCS response to intravenous insulin-has also been shown to be abnormal in some severely depressed patients and was associated with dexamethasone unresponsiveness (Carroll, 1969). Butler and Besser

*Research Fellow, National Health and Medical Research Council, Department of Psychiatry, University of Melbourne.

tCato Professor of Psychiatry, Department of Psychiatry, University of Melbourne, Clinical Sciences Building, Royal Melbourne Hospital, Melbourne, Victoria, Australia.
(1968) reported raised plasma and urinary corticosteroid levels, a disturbed diurnal rhythm, and adrenocortical resistance to dexamethasone suppression in three patients with severe depression. These abnormalities are similar to those observed in Cushing's syndrome but revert to normal with successful antidepressant treatment. A review of these and other studies in severe disorders of mood has led to the conclusions "that some severely depressed patients show an increase in adrenocortical activity and disturbances in the feedback control of A.C.T.H. release" and that "manic patients and some severely depressed patients show a normal pattern of adrenal-pituitary function" (Davies, 1969).

The present paper compares clinical and questionary data from two groups of patients admitted to hospital with a primary depressive illness, who have been separated by their plasma 11-OHCS response to dexamethasone, $10 \mu \mathrm{g} / 100 \mathrm{ml}$. being taken as the separation level.

\section{Subjects and Methods}

Fifty patients with severe depressive illnesses admitted consecutively as inpatients to the professorial psychiatric unit were studied. They were all typical melancholic patients with agitation, retardation, delusional and suicidal thoughts, as well as disturbance of sleep, appetite, and bowel function. The clinical data were obtained from patients and relatives and the presence or absence of certain clinical features of the history and mental state discussed and agreed on at a teaching session. In this regard "adverse childhood experiences" included death or prolonged absence of one or both parents before the age of 14, time spent in an orphanage or several foster homes, and gross parental discord. As regards previous personality features, attention was directed to pronounced obsessional 